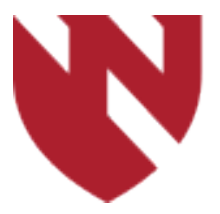

December 2019

\title{
Adherence to Practice Guidelines for Treating Diabetic Foot Infections: An Opportunity for Syndromic Stewardship
}

\author{
Randy McCreery \\ University of Nebraska Medical Center \\ Scott Bergman \\ University of Nebraska Medical Center \\ Trevor VanSchooneveld \\ University of Nebraska Medical Center
}

Tell us how you used this information in this short survey.

Follow this and additional works at: https://digitalcommons.unmc.edu/gmerj

Part of the Higher Education Commons, and the Medicine and Health Sciences Commons

\section{Recommended Citation}

McCreery, R., Bergman, S., , VanSchooneveld, T. Adherence to Practice Guidelines for Treating Diabetic Foot Infections: An Opportunity for Syndromic Stewardship. Graduate Medical Education Research Journal. 2019 Dec 13; 1(1). https://digitalcommons.unmc.edu/gmerj/vol1/iss1/24

This Conference Proceeding is brought to you for free and open access by DigitalCommons@UNMC. It has been accepted for inclusion in Graduate Medical Education Research Journal by an authorized editor of DigitalCommons@UNMC.For more information, please contact digitalcommons@unmc.edu. 
Adherence to Practice Guidelines for Treating Diabetic Foot Infections: An Opportunity for Syndromic Stewardship

\section{Creative Commons License}

\section{(c) (1) $\Theta(9$}

This work is licensed under a Creative Commons Attribution-Noncommercial-No Derivative Works 4.0 License. 


\section{Burnout in Pediatric Residents: When is Burnout Most Likely to Occur During an Inpatient Service Month and What Are its Associated Factors}

Maria Skorey, Valerie Rinehart, Kortany McCauley, Katie Greenwood, Hao Hsu

Mentor: Hao Hsu

Program: Pediatrics

Background: Burnout is a characterized by emotional exhaustion (EE), depersonalization (DP), and a lack of personal accomplishment (PA). Data shows that those in medical training suffer from burnout, but neither the timing of burnout in a narrower timeframe nor its associations are known.

Methods: A single center prospective study was conducted to establish the prevalence of burnout in pediatric residents during an inpatient month and monitor the pattern of burnout progression throughout the month and ascertain what factors may be associated with that burnout. Surveys assessing burnout, symptoms of depression, quality of life, fatigue, clinical and personal experiences were distributed to residents each week for five weeks during an inpatient service month.

Results: Mean values of EE had statistically significant worsening between the start and end of an inpatient month. Similarly, the prevalence of burnout increased from $71 \%$ to $83 \% .71 \%$ of residents had positive screens for depression. $38 \%$ of residents had answered "yes" to the question "have you doubted your career choice in the last week."
Conclusions: A large majority of pediatric residents are burned out and increases during an inpatient rotation and trigger positive screens for depression while many question their career choice during an inpatient rotation. Intervention is warranted in this center and is currently underway. Future multi-center studies are needed to validate this data, as well as follow-up studies to evaluate the possible impact of current interventions at this center.

https://doi.org/10.32873/unmc.dc.gmerj.1.1.023

\section{Adherence to Practice Guidelines for Treating Diabetic Foot Infections: An Opportunity for Syndromic Stewardship \\ Randy McCreery, Scott Bergman, Trevor VanSchooneveld}

Mentor: Trevor VanSchooneveld

Program: Infectious Disease

Background: Adherence to the Infectious Diseases Society of America (IDSA) guidelines for the treatment of diabetic foot infections (DFIs) has been associated with improved outcomes. Yet, compliance with these guidelines has been reported to be low. We initiated a quality improvement project aimed at improving guideline adherence for DFI management. Baseline results are reported here.

Methods: We reviewed all hospitalized primary DFIs newly initiated on antibiotics over 1 year (7/14-6/15). We collected demographics, DFI severity per IDSA guidelines, antibiotic use, and microbiology data. Guideline adherence for culturing and empiric antibiotic choice (based on severity) was assessed per IDSA guidelines. We then created an institutional guideline and electronic order set with built-in clinical decision support. Educational lectures on DFI best practices were given to providers who commonly treat DFIs.

Results: 177 DFI admissions were identified: $40 \%$ severe $(n=70), 47 \%$ moderate $(n=84)$, $8 \%$ mild $(n=14)$ and $5 \%$ with no evidence of infection $(\mathrm{n}=9)$. Demographics: mean age 58 years; $68 \%$ male, mean $\mathrm{HgbA1c} 8.6 \%$, length of stay 6.9 days, three-year mortality $13 \%$. Empiric antibiotic regimens were judged inappropriate in $36 \%(64 / 177)$ of cases. The most common reason for inappropriate antibiotic use was unnecessary coverage for Pseudomonas aeruginosa in 50\% (54/107) of non-severe cases. In 28\% (39/140) of cases with an ulcer, wound or skin breakdown, a superficial swab culture was obtained which is inappropriate. Only $33.3 \%(n=56)$ had a deep tissue culture obtained. In patients with deep tissue cultures, methicillin-resistant Staphylococcus aureus (MRSA) was found in $11 \%(6 / 56)$ of cases but covered for empirically in $88 \%(50 / 56)$. Pseudomonas was found in $2 \%(1 / 56)$ of cases but covered for empirically in $73 \%(41 / 56)$.

Conclusion: MRSA and Pseudomonas are uncommon DFI pathogens yet are frequently treated empirically. Inappropriate antibiotic use is often due to empiric coverage for Pseudomonas in non-severe DFIs where it is a rare pathogen. Culture practices are also less than ideal with frequent superficial swabs and underutilization of deep cultures. Institutional guidelines were developed to specifically address these issues and data collection of the impact of this project is in process. https://doi.org/10.32873/unmc.dc.gmerj.1.1.024

\section{Extraction Kinetics of Free Thyroxine with Therapeutic Plasma Exchange in the Treatment of Thyroid Storm in a Case of Antithyroid Induced Pancytopenia Ameena Madan Paramasivan, Abbey L. Fingeret, Sara M. Shunkwiler, Whitney S. Goldner}

Mentor: Whitney S. Goldner

Program: Diabetes, Endocrinology, and Metabolism

Introduction: Thyroid storm is a rare metabolic crisis associated with significant mortality. Therapeutic plasma exchange is suggested when there is a failure of conventional treatments for thyroid storm of any etiology. The efficacy of therapeutic plasma exchange is not clearly studied and currently there is no consensus available on the exact percent reduction of Free Thyroxine (FT4) that can be anticipated with each session of plasma exchange. This case illustrates the extraction kinetics of FT4 with each session and over time.

Case Presentation: We report a case of a 66 year old Caucasian female with past medical history of Graves disease in remission, recurrent hospital admissions for atrial fibrillation, and recent initiation of amiodarone, who presented with heart failure exacerbation and thyroid storm. She was started on methimazole but experienced acute pancytopenia and so the medication was discontinued. Glucocorticoids, lithium and cholestyramine were initiated but without improvement. Therapeutic plasma exchange was performed.

Results \& Discussion: Patient underwent 4 sessions of plasma exchange. FT4 measurement before and 2 hours post plasma exchange showed approximately $15-33 \%$ reduction with each session. However, FT4 rose again prior to the next day of therapy but 